\title{
Proceedings of the 135th Semon Club, 16 May 2008, Otolaryngology Department, Guy's and St Thomas' NHS Foundation Trust, London, UK
}

Chairman: Miss Elfy B Chevretton, Consultant ENT Surgeon, Guy's and St Thomas' NHS Foundation Trust.

Pathologists: Professor Leslie Michaels, and Dr Ann Sandison, University College London.

Radiologist: Dr Steve Connor, Consultant Radiologist, and Dr Daniel Bell, Specialist Registrar in Radiology, Guy's and St Thomas' NHS Foundation Trust.

Secretary: Mr Dosh Sandooram, Specialist Registrar in ENT, Guy's and St Thomas' NHS Foundation Trust.

A prize was awarded for the best presentation of the meeting, to Mr Myooran Sithamparanathan.

\section{Otology and skull base session}

Chairman: Mr Dan Jiang

Facial palsy, dysphonia, recurrent aspiration and gait imbalance but no hearing loss

H S Aw Yeang, S T Baer

From Conquest Hospital, St Leonards-on-Sea, UK.

\section{Introduction}

Neurofibromatosis type two is an autosomal dominant condition localised to chromosome 22q11, which encodes for the tumour suppressor protein schwannomin. Neurofibromatosis type two may be diagnosed using the Manchester criteria which requires either: (1) Bilateral vestibular schwannomas, or, (2) Unilateral vestibular schwannoma and/or a positive first degree relative family history of Neurofibromatosis type two, and 2 or more of the following: meningioma, glioma, neurofibroma, schwannoma, posterior subcapsular lenticular opacities.

\section{Case report}

A 26-year-old, right-handed receptionist was referred by her doctor with dysphonia. At consultation, other features which had developed over the preceding three years became apparent, including: left House-Brackmann grade two facial palsy, right vocal fold palsy, aspiration with liquids, left-sided uvula deviation, unsteady gait, bilateral upgoing plantars and a clawed left hand.

\section{Radiological findings}

Magnetic resonance imaging findings included bilateral vestibular schwannomas and multiple meningiomas. There was also a cervical cord lesion extending from the foramen magnum to $\mathrm{C} 3$ and causing significant cord compression.

\section{Management}

Following a neurosurgical assessment, the patient was admitted for urgent cervical decompression.

\section{Discussion}

Professor Leslie Michaels commented that vestibular schwannomas in neurofibromatosis type two were more aggressive compared with non-neurofibromatosis type two vestibular schwannomas, despite identical histological appearances. Furthermore, meningiomas in neurofibromatosis type two may take the form of unencapsulated collections of psammoma bodies.

\section{Conclusions and lessons learned}

Bilateral vestibular schwannomas are the hallmark of neurofibromatosis type two, but patients usually present with symptoms arising from other, associated tumours. This patient denied hearing difficulties but had several other complaints which merited individual ENT investigation. It is clinically satisfying to arrive at a single diagnosis, but for the patient it is undoubtedly devastating news.

\section{An unusual cause of vertigo in a patient with previous bilateral, radical mastoidectomies}

M Whittaker, N Patel, M Bhattacharyya

From Whipps Cross University Hospital, London.

A 46-year-old man presented to the ENT clinic with a six-week history of dizziness, otalgia, and frontal and occipital headaches with neck pain. He had a past history of bilateral, radical mastoidectomies many years ago and had been admitted the previous week for regular microsuction of his moist mastoid cavities, together with topical antibiotic and steroid eardrops therapy. He had been discharged home with persistent symptoms.

Examination showed stable mastoid cavities. It was felt that the patient's dizziness was non-otological in origin. He was referred for a medical opinion and the physicians arranged a computed tomography scan, which was reported as 'bilateral cholesteatoma with marked bony erosion'. The patient was referred straight back to ENT for admission. A finding of stable mastoid cavities was again concluded, and a further medical opinion was sought. A magnetic resonance imaging scan was arranged, which showed grossly abnormal uptake of contrast in the caudate nucleus and basal ganglia. Subsequently, the patient underwent a lumbar puncture (undertaken by the neurologists). Microscopic examination of cerebrospinal fluid confirmed cryptococcal meningitis. Analysis for human immunodeficiency virus was also positive.

The patient was treated with intravenous amphotericin, flucytosine and ceftriaxone, and responded well to treatment.

This case highlights the fact that not all vertigo is otological in origin. It also demonstrates the importance of good clinical history-taking in establishing a diagnosis, and the need to interpret radiological findings in the light of the clinical history. 
An unusual cause of congenital conductive hearing loss

S Pal, M Bhattacharyya, S Jayaraj

From Whipps Cross University Hospital, London.

Case report

A 26-year-old man presented with right-sided hearing loss. As a child he had suffered chronic otitis media with effusion, for which he had undergone repeated ventilation tube insertion procedures.

Otoscopic findings revealed a structurally normal external auditory canal and an intact, atelectatic tympanic membrane with the handle of the malleus not visible. Audiological testing revealed a moderate to severe conductive hearing loss, with a $40 \mathrm{~dB}$ air-bone gap and a type $\mathrm{A}_{\mathrm{d}}$ tympanogram. High resolution computed tomography indicated the absence of the right malleus despite the presence of the incus and stapes, and normal findings on the left.

The patient subsequently underwent exploratory tympanotomy, which confirmed the radiological diagnosis. No erosive process or cholesteatoma was found. The incudostapedial anatomy was normal. A type II tympanoplasty was performed.

\section{Discussion}

Malleolar abnormalities are relatively common in conjunction with major congenital ear atresia, but are not often described as isolated findings. This case highlights the importance of meticulous examination of all quadrants of the tympanic membrane, and the need to reconsider diagnoses in patients with ongoing symptoms.

This patient had previously undergone several procedures, during which the absence of his malleus had not been noted. Furthermore, from an early age audiology had consistently shown a $40 \mathrm{~dB}$ conductive hearing loss across all frequencies, while the patient's tympanogram had progressed from a type $B$ to a type $A_{d}$. This should have raised suspicion of ossicular discontinuity, as opposed to the presumed diagnosis of ongoing middle-ear effusions.

\section{A case of sudden, profound hearing loss secondary to a television injury}

S Fishpool, N J Holland, A F Fitzgerald O'Connor

From Guy's and St Thomas' NHS Foundation Trust, London.

\section{Case report}

A 27-year-old student presented with acute, left-sided deafness, in her only hearing ear, after sustaining a glancing blow from a falling television. There had been no loss of consciousness, and she had used the telephone in the affected ear immediately after the event. There was a history of profound deafness in the right ear dating from childhood. Examination and audiometric assessment showed severe, bilateral, sensorineural hearing loss (SNHL) and a minor skin abrasion.

\section{Radiological findings}

Computed tomography (CT) head scans excluded a temporal bone fracture. Fine-cut $\mathrm{CT}$ and magnetic resonance imaging of the temporal bones was reported by Dr Steve Connor as confirming bilateral widened vestibular aqueducts.

\section{Management}

The patient was admitted for bed rest and intravenous steroids. Over five days, her left-sided SNHL reduced from 90 to $65 \mathrm{~dB}$ (averaged over the speech frequencies). The patient demonstrated increasing benefit from a leftsided hearing aid.

\section{Conclusion and lessons learned}

'Large vestibular aqueduct syndrome' is classically associated with sensorineural hearing loss caused by minor head trauma, which produces a stepwise deterioration with incomplete recovery. Mr Holland referred to the published findings of the House group and others, which reported that surgical attempts to close the dilated aqueduct(s) had been unsuccessful. Mr Jiang and Mr Holland reported that, in the experience of the St Thomas' auditory implant team, patients referred with large vestibular aqueduct syndrome often performed well with hearing aids, and cochlear implantation was successful when hearing declined further. Professor Michaels noted that there was an association with Pendred's syndrome. However, this patient did not have a clinically apparent goitre.

\section{Severe, relapsing otitis externa in a child requiring surgical intervention}

N J Holland, M J Gleeson

From Guy's and St Thomas' NHS Foundation Trust, London.

Case report

A two-and-a-half-year-old child had suffered from continual, bilateral ear infections from eight months of age. The infections had been resistant to topical and systemic antibiotics and had resulted in extensive destruction of the external auditory canals and bilateral central perforations. Over a two-year period, the child had required more than 40 general anaesthetics for microsuction, wick placement and mastoid exploration. A detailed immunological evaluation did not identify any immunodeficiency.

\section{Radiological appearance}

Two computed tomography scans, taken eight months apart, showed progressive erosion of the bony external auditory canals without significant mastoid disease.

\section{Management}

The child underwent left-sided, subtotal petrosectomy with cochlear preservation and blind-sac closure of the external auditory canal. There was dense tympanosclerosis with evidence of new bone formation adjacent to the otic capsule. There were no complications. The child remained well after surgery, and fitting of a softband-mounted, bone-anchored hearing aid assisted satisfactory speech and language development.

\section{Histology}

Histological examination of the skin of the external auditory canal showed chronic, non-specific inflammation with a moderate lymphocytic infiltrate, granulation tissue and a foreign body reaction to keratin.

\section{Conclusion and lessons learned}

Professor Leslie Michaels felt that, although there was some evidence of a keratin foreign body reaction, the condition was unlikely to represent a congenital disorder of keratin migration. An acquired, necrotising otitis externa, injuring the normal external canal epithelium, was felt to be the cause. Mr Holland raised the option of a future bone-anchored hearing aid or middle-ear implant (floating mass transducer to round window) for hearing rehabilitation. 


\section{Paediatric session}

Chairman: Mr Gavin Morrison

\section{Persistent nasal obstruction in a child}

S Pal, M Whittaker, M Patel

From Whipps Cross University Hospital, London.

Case report

A 12-year-old girl presented with a history of chronic nasal obstruction and rhinorrhoea. Her general practitioner had been treating her for presumed allergic rhinitis with beclomethasone and ipratropium nasal sprays for five years, without any benefit. Anterior rhinoscopy in the ENT clinic showed bilateral, hypertrophic inferior turbinates with a rhinitic nasal mucosa and copious secretions, which were more prominent on the left side.

Initial radiological findings

An X-ray of the post-nasal space showed enlarged adenoids.

\section{Management}

The patient was treated subsequently for two years with repeated courses of betamethasone drops, fluticasone spray and oral antihistamines, without benefit. On further review, nasal endoscopy showed a mass of granulation tissue alongside the left middle turbinate.

\section{Further radiological findings}

A computed tomography scan confirmed a calcified rhinolith in the left nasal cavity extending into the post-nasal space.

\section{Further management}

The patient underwent endoscopic rhinolith removal, assisted with a microdebrider, together with suction diathermy adenoidectomy. She made an uneventful recovery.

\section{Conclusion and lessons learned}

This case highlights the importance of a thorough nasal examination in children with persistent nasal symptoms despite aggressive medical treatment. The enlarged adenoids in this case somewhat masked the classical findings of unilateral rhinorrhoea as the presenting feature of a nasal foreign body. It is important that endoscopic evaluation is always performed in children with unilateral, chronic, purulent nasal discharge.

\section{Solitary, persistent submandibular lymph node enlargement in a nine-year-old}

W Lee, M Moonim, I Hore

From Guy's and St Thomas' NHS Foundation Trust, London.

\section{Introduction}

Persistent cervical node enlargement should always raise the suspicion of lymphoma. However, rare, reactive causes can mimic lymphoma. Excision biopsy is usually the 'gold standard' investigation.

Case report

A nine-year-old boy presented with an asymptomatic, $2 \mathrm{~cm}$, firm, smooth, left submandibular node which had been present for three months. The rest of the examination was normal, as was an infection screen and a chest $\mathrm{X}$-ray. The parents were not keen on an excision biopsy. An ultrasound scan with fine needle aspiration cytological examination showed features suspicious of lymphoma.
However, flow cytometry of the fine needle sample found the cells to be polyclonal, which would suggest a reactive node. After a second ultrasound scan showed features suspicious of lymphoma, the parents agreed to an excision biopsy.

\section{Histological findings}

Initial inspection suggested Castleman's disease, but an expert opinion diagnosed 'progressive transformation of germinal centres, with regressed germinal centres. No lymphoma'.

\section{Management}

The patient was followed up for any recurrence.

\section{Conclusions and lessons learned}

Progressive transformation of germinal centres together with regressed germinal centres in a cervical node is an extremely rare form of reactive lymph node enlargement which can mimic lymphoma clinically, ultrasonographically and cytologically. Flow cytometry can be helpful in demonstrating polyclonal lymphocytes (consistent with a reactive condition) rather than monoclonal lymphocytes (consistent with lymphoma), although the possibility of a sampling error should always be borne in mind. The histology of the condition is complex and expert opinion is required for final diagnosis. Excision is the management of choice for diagnosis and treatment.

\section{Right nasal mass in a 20-month-old girl}

C Patel, K Edmonds, I Hore

From Evelina Children's Hospital, Guy's and St Thomas' NHS Foundation Trust, London.

Introduction

The differential diagnosis of a nasal mass is large, and includes the possibility of a mass of intracranial origin.

\section{Case report}

A 20-month-old girl presented with a history of right-sided nasal obstruction present from birth. The rest of the examination was normal. Cystic fibrosis and primary ciliary dyskinesia tests were negative. Examination under anaesthesia revealed a right nasal mass. Magnetic resonance imaging (MRI) was initially reported as showing a partially cystic mass communicating with the right frontal lobe through the cribriform plate, suggesting a possible diagnosis of anterior fossa encephalocoele. Subsequent computed tomography (CT) scanning revealed no connection between the mass and the cranial cavity. At the time of ENT referral, at 22 months, the child's nasal obstruction had started to improve. She underwent endoscopic biopsy and debulking of the lesion.

\section{Radiological findings}

A review of the original MRI at the Semon Club demonstrated the mass to be confined to the nasal cavity, with no enhancement of the dura or distortion of the crista galli. In retrospect, the CT scan was of no additional value.

\section{Histological findings}

Histological examination of the biopsy showed astrocytes within a background of fibrous tissue, confirming a diagnosis of nasal glioma.

\section{Management}

Endoscopic removal of the lesion, down to macroscopically normal tissue, was performed. At follow up, the patient remained asymptomatic. 


\section{Conclusion and lessons learnt}

(1) Magnetic resonance imaging is the investigation of choice, rather than CT, in children of this age group, due to insufficient ossification. (2) Nasal gliomas may involute. (3) Endoscopic surgery can achieve adequate clearance and a good cosmetic result.

\section{Rapidly enlarging craniofacial mass with left-sided proptosis}

O Ajayi, M Soma, B Hartley

From the Great Ormond Street Hospital for Children, London.

\section{Introduction}

We present a child who presented late with an extensive, disfiguring facial mass, impaired vision and dysphagia.

\section{Case report}

A 19-month-old girl was referred with a large, left-sided facial mass which affected her airway, swallowing, left eye and left external auditory canal. The adjacent skin was hyperpigmented, with multiple café-au-lait spots. She had previously undergone a gastrostomy and tracheostomy abroad, having been advised that the mass was inoperable.

\section{Radiological findings}

Magnetic resonance imaging scans were in keeping with a massive plexiform neurofibroma involving the left side of the face, neck, tongue, infratemporal fossa and all the deep fascial spaces. There was marked narrowing of the oropharynx. In addition, dysplasia of the left sphenoid wing was noted. There was also orbital and intracranial extension with involvement of the left trigeminal nerve.

\section{Histological findings}

Histology showed a lobulated, myxoid, spindle cell lesion with a plexiform architecture, consistent with a plexiform neurofibroma.

\section{Management}

The multidisciplinary team's decision was to offer a subtotal resection without adjuvant therapy. The child's recovery was complicated by poor wound healing, which necessitated tissue debridement and management by the plastic surgery team.

\section{Conclusion}

At the time of writing, the patient's prognosis was unknown. The decision to operate was made based on the lesion's intracranial and orbital involvement and growth rate.

\section{Nasal obstruction in a child with lytic lesions of the phalanges}

P O Pal, A J Donne, G Morrison

From the Evelina Children's Hospital, Guy's and St Thomas' NHS Foundation Trust, London.

\section{Introduction}

Nasal obstruction in a child is common, but its association with obvious facial deformity is rare. The differential diagnosis is limited, and the final diagnosis invariably requires radiological investigation and histological examination.

\section{Case report}

An eight-year-old boy was referred to the ENT department with left-sided nasal obstruction. He had a history of Noonan's syndrome, facial dysmorphism, pulmonary artery stenosis, primary right renal agenesis and primary hyperparathyroidism. A computed tomography (CT) scan was performed and a nasal polyp was excised. Two years later, he re-presented with total nasal obstruction. Examination showed oedematous nasal septal mucosa with a cobblestone appearance. His tonsils were grossly enlarged. He also had subcutaneous lesions overlying the nasal bones. A sleep study demonstrated significant obstructive sleep apnoea. He underwent a tonsillectomy and biopsy of the friable nasal septal mucosa.

\section{Radiological findings}

Facial CT scanning indicated a $3 \times 6 \mathrm{~mm}$, polypoidal lesion in the left nasal cavity, arising from the septal cartilage. Previous foot and hand radiographs had shown lytic lesions in the third and fifth phalanges bilaterally, well before any facial dysmorphism had become apparent.

\section{Histopathological findings}

Macroscopically, the polyp had a dark centre. Microscopically, the polypoid nodule was covered by epidermis and a mixture of giant cells within a vascular stroma and lamellar bone.

\section{Management}

An endoscopic nasal polypectomy was performed. At the time of writing, the lesions arising from the nasal bones were planned to be excised using an open rhinoplasty technique.

\section{Conclusion}

Noonan's syndrome is very rarely associated with giant cell lesions, which can be managed with conservative surgical intervention.

\section{Rhinology session}

Chairman: Mr David Roberts

\section{A rare tumour of the nasal ala}

S Fishpool, D Gillett, D Roberts

From Guy's and St Thomas' NHS Foundation Trust, London.

\section{Case report}

An 18-year-old man presented to the rhinology clinic with a three-year history of a slow-growing, right-sided alar mass, clinically resembling a pyogenic granuloma. His past medical history was significant for a mitochondrial cytopathy and related learning difficulties. A shave excision of the lesion was performed under general anaesthesia.

\section{Histological findings}

Histological examination required dermatopathological review, and was classified as a low grade trichoblastic/trichilemmal carcinoma. Complete excision was recommended. Professor Leslie Michaels explained that these lesions behave clinically like basal cell carcinomas.

\section{Management}

Due to the patient's learning difficulties and needlephobia, the general anaesthetic administered for the shave excision was challenging for all involved. At the time of writing, a computed tomography scan of the neck followed by a wide local excision of the lesion and insertion of titanium fixture abutments was scheduled under a single general anaesthetic. The abutments would allow the fitting of an appropriate prosthesis which could be removed as required during follow-up examinations. Mr Howard, from the Royal National Throat, Nose and Ear Hospital, 
London, suggested that a bi-lobed right cheek rotation flap may be an alternative cosmetic option.

\section{Conclusion and lessons learned}

Trichoblastic or trichilemmal carcinomas of the nose are rare. This is the first mixed trichoblastic/trichilemmal case described. Clinicians should be aware of the diagnosis of trichilemmal and/or trichoblastic carcinoma in the differential diagnosis of masses arising from the nasal ala of patients of all ages.

\section{An unusual cause of complete nasal obstruction}

M Whittaker, S Pal, S Jayaraj

From Whipps Cross University Hospital, London.

\section{Case report}

A 76-year-old man presented with a 12-month history of complete nasal obstruction, intermittent right-sided epistaxis, anosmia and left epiphora. Rhinoscopy revealed friable, grade III nasal polyps bilaterally.

\section{Radiological findings}

A computed tomography scan showed extensive involvement of the frontal, sphenoid and particularly ethmoid sinuses, with abnormal invasion of the orbits and medial recti.

\section{Management}

The aims of the initial surgical intervention were to provide an adequate nasal airway and to obtain tissue for diagnosis. Endoscopic debulking of the polypoidal tissue was therefore performed.

\section{Histological findings}

Histological examination revealed an olfactory neuroblastoma, confirmed by immunohistochemical analysis. According to the four-tiered Hyam's grading system, this case was categorised as grade three.

\section{Further management}

The patient subsequently underwent a craniofacial resection with the intent of surgical cure.

\section{Discussion}

Malignant tumours of the nasal cavity are rare, and only 6 per cent are olfactory neuroblastomas. These lesions can be easily missed as presenting symptoms mimic those of benign tumours. Since olfactory neuroblastomas are so uncommon, strategies for management are a matter of contention. Treatment recommendations range from a minimally invasive approach to craniofacial resection combined with radiotherapy.

\section{Malignant transformation of Schneiderian inverted papilloma in sinonasal region}

S M Keh, T Jacob, N Salama

From University Hospital Lewisham, London.

We presented two cases which we initially thought were Schneiderian inverted papillomas in the sinonasal region; however, when the histological slides were reviewed at the meeting by Professor Leslie Michaels and Dr Ann Sandison, the lesions were thought more likely to be squamous cell carcinomas.

According to Professor Leslie Michaels, malignant transformation of inverted papillomas in the sinonasal region is rare and estimated to occur in the region of 1-2 per cent, despite current literature suggesting a rate of 5-15 per cent. It is possible that some of these cases were misdiagnosed squamous cell carcinomas.

The quality of literature available on inverted papillomas in the sinonasal region varies. It is important for ENT trainees to be able to critically appraise published papers and to use their clinical judgement in order to assess each case individually.

When completing the histopathology request form, it is important to include a brief description of the clinical history, examination findings and radiological features.

Finally, inverted papillomas should be routinely discussed at the head and neck multidisciplinary team meeting, not only because of their aggressive nature and their ability to transform into squamous cell carcinomas, but also to confirm that the biopsy does not show features of squamous cell carcinoma. If the histological findings do not correspond with the clinical and radiological features, a second opinion should be sought from a histopathologist who is familiar with sinonasal tumours.

\section{A rare case of recurrent epistaxis}

B Fu, E Koury, J Marais

From Northwick Park Hospital, Harrow, UK.

\section{Introduction}

We present a patient with an undiagnosed sinonasal tumour. She had been operated upon via two different surgical approaches, 12 years apart, with significant differences in associated morbidity.

\section{Case report}

A 53-year-old woman presented with a history of recurrent, left-sided epistaxis 12 years previously. She had undergone a procedure combining lateral rhinotomy, radical external ethmoidectomy and sphenoidectomy, in order to remove an undiagnosed sinus tumour. This procedure had left her with a large facial scar. She had remained asymptomatic until she re-presented with a history of recurrent, left-sided epistaxis in recent weeks.

\section{Radiology}

Computed tomography and magnetic resonance imaging showed a left, sphenoidal, heterogeneously-enhancing, soft tissue mass extending anteriorly into the sphenoethmoidal recess.

\section{Management}

Resection of the recurrent tumour was performed via functional endoscopic sinus surgery. The tumour pedicle was debrided from the lateral wall of the sphenoid sinus, above the internal carotid artery. The patient was discharged home uneventfully on the first post-operative day.

\section{Histopathology}

On immunostaining, the spindle cells were negative for cytokeratin marker AE1/3, vascular markers (CD34 and CD31), desmin, S100, CD45 and CD117. The cells showed reactivity with vimentin, SMA, FXIII and BCL2. This was an unusual spindle cell lesion of the nasal sinus. The differential diagnosis included: haemangiopericytoma, cellular haemangioma, angiofibroma, solitary fibrous tumour, glomus tumour and smooth muscle tumours. Professor Leslie Michaels agreed with these findings and suggested that the appearances and immunoprofile were mostly in keeping with a sinonasal-type haemangiopericytoma. 


\section{Conclusion}

The diagnosis and optimal management of haemangiopericytoma remain clinical challenges for otorhinolaryngologists. Although this type of tumour shows no malignant features, long term follow up is essential for disease surveillance.

\section{An unusual mass in the right nasal cavity: a diagnostic dilemma for all!}

A Joshi, J P Jeannon

From Guy's and St Thomas' NHS Foundation Trust, London.

Case report

A 45-year-old woman of Bangladeshi origin presented with an ulcerating mass in the right nasal cavity and referred otalgia. Examination under anaesthesia of the nose revealed multifocal, friable, polypoidal lesions in the right nostril extending to the anterior end of the middle turbinate. Biopsies were taken for histological and microbiological examination. Cultures revealed a moderate growth of klebsiella species.

\section{Histology}

Initial histological analysis showed features suggestive of plasmacytoma or myeloma. However, further investigations were normal, including liver profile, bone profile, immunoglobulin M levels, protein electrophoresis and paraprotein levels. A review of the histological slides with periodic acid Schiff staining revealed the presence of organisms within macrophages. A diagnosis of rhinoscleroma was made.

\section{Management}

The patient was prescribed a six-week course of ciprofloxacin. The lesions regressed significantly.

\section{Discussion}

Rhinoscleroma is a chronic, granulomatous infection caused by Klebsiella rhinoscleromatis, which is rare in the developed world. However, it should be considered in patients originating from endemic countries. There are three stages: catarrhal, granulomatous and sclerotic. Histological features include large, vacuolated Mikulicz cells and transformed plasma cells with Russell bodies. Antibiotic therapy is the treatment of choice, and surgical debridement is occasionally required.

In the UK, rhinoscleroma is an extremely rare disorder of the upper aerodigestive tract. Professor Leslie Michaels agreed that it frequently posed a diagnostic dilemma and could be difficult to differentiate from neoplastic conditions such as plasmacytoma.

\section{Head and neck session}

Chairman: Mr Jean-Pierre Jeannon

\section{Exophytic lip lesion with dark discolouration of gingival and buccal mucosa}

J Joseph, J Stephens, A Thorpe

From the West Middlesex University Hospital, London.

\section{Introduction}

Malignant melanoma of the head and neck is rare, and can present in a variety of ways. No record of an association of this condition with acanthosis nigricans could be found.

Case report

We present a case of a 51-year-old man who presented via his general practitioner with a large, exophytic lesion in association with a discolouration of the gingival and buccal mucosa characteristic of acanthosis nigricans. He was a heavy smoker and had a history of alcohol abuse. A tumour measuring $2 \times 2 \times 1.5 \mathrm{~cm}$ was excised from his lip.

\section{Histology}

Histological examination of the lesion revealed it to be a nodular, amelanotic, malignant melanoma in the vertical growth phase. There was no lymphovascular invasion and there were clear margins of $3 \mathrm{~mm}$

\section{Radiology}

A staging computed tomography scan demonstrated no metastatic spread of disease.

\section{Management}

No further treatment was necessary as the tumour had been completely excised.

\section{Comments}

Mr David Howard stressed the importance of distinguishing cutaneous malignant melanoma of the head and neck, as in this case, from those involving the mucosae. Grading for cutaneous malignant melanoma involves using Clark's level and Breslow thickness. These are not helpful for mucosal malignant melanoma.

Acanthosis nigricans is a known external marker of internal malignancy. We suggest that this is the first report of this hyperpigmentation disorder occurring in association with an oral cavity melanoma, and an interesting example of an unusual diagnosis.

\section{Intrathyroid air}

S Haikel, M Black, D Mitchell

From the William Harvey Hospital, Willesborough, UK.

\section{Case report}

A 64-year-old lady presented with a one-month history of increasing inspiratory stridor, weight loss and anterior neck swelling. Flexible nasendoscopic examination of the supraglottis and glottis was normal.

\section{Radiological findings}

A large soft tissue mass was found to extend from the lower pole of the left lobe of the thyroid into the retrosternum, displacing the trachea to the right. Air was noted within the left thyroid lobe.

\section{Management}

The patients' stridor initially improved with corticosteroids, antibiotics and humidified oxygen. Within 10 days, however, the disease had progressed, with increasing stridor. The patient gave consent for panendoscopy, bronchoscopy and total thyroidectomy with level six neck clearance. At bronchoscopy, the tracheal lumen was found to be infiltrated by tumour, with exposed cartilaginous rings. A tracheostomy was therefore performed and tissue sent for histological analysis. The remainder of the procedure was abandoned.

\section{Histological findings}

An initial fine needle aspiration cytology examination had suggested malignancy. Paraffin sections of the operative specimen showed a poorly differentiated squamous cell carcinoma. Following discussion at the head and neck multidisciplinary team meeting, it was felt that palliation with chemoradiotherapy was appropriate. The patient died, secondary to airway obstruction, prior to the commencement of chemoradiation. 


\section{Conclusion and lessons learned}

Bronchoscopic examination of the airway is mandatory in all patients presenting with stridor and a neck mass. $\mathrm{Mr}$ David Howard suggested that an alternative management option might have been referral to another centre for consideration of palliative tracheal stenting.

\section{Chondrosarcoma of the larynx}

P Modayil, T Odutoye, P A Williamson

From St George's Hospital, London.

\section{Introduction}

Chondrosarcomas are rare tumours accounting for less that 1 per cent of all laryngeal tumours. We present two cases of laryngeal chondrosarcoma.

\section{Case report}

Case A was a 72-year-old man who presented with a two-year history of dysphonia and worsening shortness of breath. Case B was an 84-year-old man who had been diagnosed with non-small-cell carcinoma of the lung; his chest physician had referred him to the head and neck surgeons as the patient's left vocal fold had appeared bulky on bronchoscopy.

\section{Radiological findings}

A computed tomography (CT) scan in case A showed a large posterior laryngeal mass arising at the level of the vocal folds with subglottic extension. A CT scan in case B showed a left glottic mass with subglottic extension. Both cases showed cartilage destruction and tumour calcifications.

\section{Histological findings}

Both cases revealed features of grade two chondrosarcoma.

\section{Management}

After a multidisciplinary team discussion, case A underwent a wide-field total laryngectomy and received adjuvant post-operative radiotherapy. In case B, a tracheostomy was performed and palliative radiotherapy administered for the lung cancer.

\section{Conclusion}

Surgical excision is the treatment of choice for chondrosarcoma. There is no demonstrable benefit of adjuvant radiation or chemotherapy, although post-operative adjuvant radiotherapy is frequently given. In cases unfit for surgery, the options include laser debridement and tracheostomy to relieve airway obstruction.

\section{Comments}

Because of their slow growth and low-grade malignancy, a wait-and-watch policy and conservation laryngeal surgery are alternative options.

\section{Nasal malignant round cell tumour}

M Sithamparanathan, J Weir, A D Mace

From the Charing Cross and Hammersmith Hospitals NHS Trust, London.

\section{Introduction}

The authors present a rare case of a maxillary alveolar tumour which presented considerable histological and radiological diagnostic difficulty.

Case report

A 61-year-old woman presented with a three-month history of a painless swelling around the right nostril. On examination, there was a $2 \mathrm{~cm}$, smooth, subcutaneous swelling involving the right nasal ala, extending into the upper lip and the floor of the nose. An initial punch biopsy showed nodular elastosis.

\section{Radiological findings}

Computed tomography (CT) demonstrated a $5 \mathrm{~cm}$ mass centred on the alveolar bone of the right maxilla with spiculated calcification. There was extensive bony destruction extending into the tooth-bearing portion of the bone, the vomer and hard palate, with no pathological neck nodes. Magnetic resonance imaging showed heterogeneous signal intensity. The clinical working diagnosis was chondrosarcoma.

\section{Histological findings}

Histology findings were equivocal. Repeat biopsies showed fragments of bone infiltrated by a malignant small round cell tumour with a high mitotic count. The morphology and immunoprofile suggested a differential diagnosis of monophasic synovial sarcoma or Ewing's sarcoma/peripheral primitive neuroectodermal tumour.

\section{Management}

The patient was referred to Dr Anna Cassoni at the University College Hospital sarcoma unit for further management.

\section{Conclusion}

Monophasic synovial sarcoma and Ewing's sarcoma/peripheral primitive neuroectodermal tumour have only rarely been described at this site. The treatment protocol for each of these tumours is different. This case demonstrates the fact that occasionally, even after representative tissue biopsy, a diagnosis cannot be reached. However, with the close cooperation of the multidisciplinary team, investigations and treatment can be successfully targeted.

\section{Malignant transformation following pharyngeal pouch stapling}

S Patil, M Z Farag

From Chase Farm Hospital, Middlesex, UK.

\section{Introduction}

Carcinoma arising in a pharyngeal pouch is rare, but the real, population-based incidence remains unknown. Although endoscopic stapling is a less invasive treatment than external excision, we should be mindful of the long term risk of malignant change.

\section{Case history}

An 84-year-old woman presented with dysphagia to solids and liquids. She had undergone endoscopic stapling of a pharyngeal pouch 10 years previously. She also had a past history of breast carcinoma. A positron emission tomography scan performed in 2006 to investigate painful ribs had shown increased uptake in the oesophageal region at the level of T1. This had been attributed to inflammation as the patient had been taking risedronate sodium for osteoporosis. Flexible nasolaryngoscopy showed no abnormality of the upper aerodigestive tract. Oesophagoscopy under general anaesthetic showed an exophytic post-cricoid tumour extending into the upper oesophagus.

\section{Radiology}

Barium swallow showed severe pharyngoesophageal narrowing between $\mathrm{C} 4$ and C6. A computed tomography 
scan revealed an infiltrating mass, presumably arising from the upper oesophagus.

\section{Histopathology}

Histological examination showed an invasive, undifferentiated squamous cell carcinoma.

\section{Management}

After discussing the management options and prognosis, the patient decided against further treatment.

\section{Conclusion and lessons learned}

There is a small but definite risk of malignancy arising in a pharyngeal pouch treated with stapling. The National Institute for Clinical Excellence 2003 guidelines recommend endoscopic stapling as a safe and noninvasive procedure for hypopharyngeal diverticulae. However, no recommendation has been made regarding post-operative follow up. We question whether there ought to be specific guidelines regarding long term follow up and surveillance for malignancy following this procedure. 